\title{
Survival outcomes for patients with four treatments in stages I-III esophageal squamous cell carcinoma: a SEER analysis
}

\author{
Jingyang Wu, Jiansheng Yang, Xianbin Lin, Liang'an Lin, Wentan Jiang, Chengke Xie \\ Department of Thoracic and Cardiovascular Surgery, The Second Affiliated Hospital of Fujian Medical University, Quanzhou, China \\ Contributions: (I) Conception and design: C Xie; (II) Administrative support: J Yang; (III) Provision of study materials or patients: W Jiang; \\ (IV) Collection and assembly of data: X Lin; (V) Data analysis and interpretation: J Wu; (VI) Manuscript writing: All authors; (VII) Final approval of \\ manuscript: All authors. \\ Correspondence to: Chengke Xie. Department of Thoracic and Cardiovascular Surgery, The Second Affiliated Hospital of Fujian Medical University, \\ Zhongshan North Road, Licheng District, Quanzhou 362000, China. Email: 18559305202@163.com.
}

\begin{abstract}
Background: Esophageal cancer (EC) is globally acknowledged as one of the most common malignancies among all gastrointestinal cancers. Furthermore, in Eastern Asia, squamous cell carcinoma is the main pathological type of EC. There are different treatments for esophageal squamous cell carcinoma (ESCC), but there is still a lack of large-sample analysis of prognosis among different treatments, especially for different tumor stages. The analysis of the prognosis of ESCC patients with different treatments may be helpful to choose the treatment methods for different stages ESCC.

Methods: A total of 3,346 patients with pathological ESCC between 1976 and 2016 were derived from the Surveillance, Epidemiology, and End Results (SEER) database. All clinical factors associated with prognosis were collected and analyzed to achieve the difference of prognosis among different treatments in ESCC patients, such as ages, sex, race, tumor grade, anatomic location and so on. Kaplan-Meier and Cox proportional hazard analysis were used to compare survival of different treatments in ESCC patients with stage I-III.
\end{abstract}

Results: The overall survival (OS) in all ESCC patients who had received surgery and surgery plus radiation therapy or/and chemotherapy are superior than that had not received any treatments and radiation therapy or/and chemotherapy. The OS in ESCC patients with stage I who had received surgery and surgery plus radiation therapy or/and chemotherapy are superior than that had not received any treatments and radiation therapy or/and chemotherapy. The OS in ESCC patients with stage II/III who had received surgery and surgery plus radiation therapy or/and chemotherapy are superior than that in other groups. Age, race and grade as an independent predictive factor for survival $(\mathrm{P}<0.05)$. A nomogram model was constructed to show surgery group had better 1-, 3- and 5-year OS than radiation therapy or/and chemotherapy group (OS: $78.5 \%$ vs. $59.2 \%, 37.9 \%$ vs. $18.4 \%, 16.9 \%$ vs. $6.1 \%$ ).

Conclusions: Surgery is still the first choice for all ESCC patients with stage I-III. Radiotherapy and chemotherapy could improve the survival rate in ESCC patients with stage II-III who have received surgery.

Keywords: Esophageal cancer (EC); therapy; prognostic; survival analysis; Surveillance, Epidemiology, and End Results (SEER)

Submitted Oct 07, 2020. Accepted for publication Mar 26, 2021.

doi: $10.21037 /$ tcr-20-2995

View this article at: http://dx.doi.org/10.21037/tcr-20-2995

\section{Introduction}

Esophageal cancer (EC), a kind of refractory tumor with poor prognosis, which ranks sixth in terms of overall mortality in cancer globally (1). The 5-year survival rate of patients with EC was $20.9 \%$ (2). The main pathotypes of EC includes esophageal adenocarcinoma (EAC) and 
esophageal squamous cell carcinoma (ESCC) (3). And ESCC is the main pathological type of EC in Eastern Asia (4). According to the guidelines of National Comprehensive Cancer Network (NCCN), endoscopic resection of early EC without lymph node lesions is recommended as an alternative to esophagectomy (5). For locally advanced ESCC without distant metastasis, NCCN recommends the neoadjuvant treatment of definitive concurrent chemoradiotherapy (CCRT), surgery or esophagectomy alone (6). The neoadjuvant chemoradiotherapy has recommended as a standard treatment in parts of Europe and the USA (7). While neoadjuvant chemotherapy has recommended as true treatment in the UK and Japan (8). However, it is generally accepted internationally that surgery remains a dominant part of the curative treatment of locally advanced EC (9). Several research reports have shown that if patients with ESCC receive chemotherapy or radiotherapy, the survival prognosis can be improved $(10,11)$. Prognostic factors of ESCC mainly include two parts. Firstly, clinical prognostic factors: several studies have shown a lack of prognostic significance related to body mass index, tobacco, age $(12,13)$. While, malnutrition, location of tumor and sex can be used as an independent prognostic factor of ESCC $(14,15)$. Secondly, histological prognostic factors: tumor stage, histological type and nodal invasion are major factors in the prognosis of EC, respectively (16-18).

There is still a lack of large-sample analysis of prognosis among different treatments, especially for different ESCC tumor stages. So, it is particularly important to compare the prognosis of different treatment in each stage of ESCC rely on the extensive sample database.

The Surveillance, Epidemiology, and End Results (SEER) Program is a National Funded Cancer Institute (NCI), and collects information from various locations and sources in specific geographies within the United States. SEER provides information on the incidence rate, staging and survival data, and clinical data since 1973 (19). Importantly in the investigation of ESCC, the SEER database includes a nearly $90 \%$ follow-up rate and records, demographic variables, tumor characteristics and survival.

So, we examined four treatments in ESCC mortality and survival trends using the SEER 18 data on people who diagnosed with epithelial ESCC during 1975-2016. We also aim to identified prognostic factors unique to overall survival (OS). We present the following article in accordance with the STROBE reporting checklist (available at http://dx.doi.org/10.21037/tcr-20-2995).

\section{Methods}

\section{Search strategy and study population}

Firstly, a number of patients with a confirmed diagnosis of ESCC were screened out from the SEER database from 1975 to 2016. The cohort was composed of unidentified individuals in the NCI SEER 18 Registry [SEER*Stat Database: Incidence-SEER 18 Custom Data (with additional treatment fields) Nov 2018 Sub]. Patients were organized by age, race, gender, tumor staging and treatments. The following data from each patient were collected for analysis: race, gender, age, differentiation grade, $\mathrm{T}$ stage, $\mathrm{N}$ stage, $M$ stage, therapy, survival data and the cause of death. The study was conducted in accordance with the Declaration of Helsinki (as revised in 2013).

In this study, the inclusion criteria were: one primary tumor only, complete data on age, race, gender, active follow-up and with more than 1 month of survival. With the exclusive cases of an unknown diagnostic source information, non-squamous cell carcinoma, incomplete TNM classification system (AJCC, 7th edition) and distant metastasis of cancer, there were still 3,346 cases for this study.

\section{Staging}

The stages of all patients were divided into stage I, II, or III disease, according to the AJCC (7th edition) criteria. Furthermore, the treatments were reported in four categorical variables (none, surgery, radiation therapy or/ and chemotherapy and surgery plus radiation therapy or/ and chemotherapy) for each reporting data.

\section{Statistical analysis}

Statistical analyses were applied with $\mathrm{R}$ software version 4.0.2 and SPSS software version 22.0 (IBM, SPSS Statistics, Chicago, IL, USA). Numerical data were expressed as mean \pm standard deviation or median for quantitative variables analyzed using one-way ANOVA. Continuous variables were first transformed into categorical data, and categories were described as frequencies and percentage and then compared with Chi-square test. With univariate and multivariate Cox proportional hazards regression analyses, independent prognostic factors were identified. In addition, survival curves were depicted by the Kaplan-Meier method and compared by the log-rank test to identify the hazard 
ratio (HR) and their 95\% confidence interval (95\% CI). $\mathrm{HR}>1$ indicates that prognostic factors are associated with decreased survival rate $(20,21)$. The $\mathrm{P}$ value $<0.05$ was confirmed as statistical significance.

\section{Results}

\section{Baseline demographic characteristics}

A total of 3,346 patients in the SEER database was collected that met the screening criteria. The baseline characteristics and different therapy of all subjects identified by the SEER database are shown in Table 1. With 60-year-old as the dividing point, the distribution of young interval and old interval is approximately accurate. The majority of patients $(66.4 \%)$ were white, $23.1 \%$ were black and $10.5 \%$ were other. The ratio of males to females was about 1.6:1, and $61.7 \%$ were male while $38.3 \%$ were female. About 781 patients (23.3\%) with stage I, 1,107 (6.2\%) with stage II, and 1,458 (43.6\%) with stage III. In terms of therapy, the surgery plus radiation therapy or/and chemotherapy proportion were greater $(16.2 \%)$ than pure surgery population $(5.8 \%)$. On the total 3,346 patients, 736 (22.0\%) did not undergo any therapy. The key prognostic factors and baseline characteristics for patients in different therapy including age, race, primary tumor site, TNM stage, tumor grade, which were summarized in Table 1.

\section{Effect of different therapy on OS}

The effect of therapeutic measures on OS was examined. Comparing the survival distributions in four groups (none, surgery, radiation therapy or/and chemotherapy and surgery plus radiation therapy or/and chemotherapy), the result showed that surgery and surgery plus radiation therapy or/and chemotherapy were clearly superior to none and radiation therapy or/and chemotherapy. Then the Kaplan-Meier survival analysis for OS based on therapeutic measures for each stage of disease were analyzed. For stage I patients, surgery and surgery plus radiation therapy or/and chemotherapy were better than the others. For stage II patients, surgery plus radiation therapy or/and chemotherapy were the most effective therapy method. the therapeutic effects of pure surgery and pure radiation therapy or/and chemotherapy were similar. For stage III patients, in terms of survival time, the first choose of therapy was surgery plus radiation therapy or/and chemotherapy, while the second was radiation therapy or/ and chemotherapy, surgery was third therapy, as shown in Figure 1.

For OS, as shown in Table 2, the mean survival time (with $95 \%$ CI) was $32 \pm 1.6$ (95\% CI: 28.6-34.9) months for none, $58 \pm 2.5$ (95\% CI: 53.1-63.1) months for surgery, $42 \pm 0.9$ (95\% CI: 40.8-44.6) months for radiation therapy or/and chemotherapy, and $57 \pm 1.5$ (95\% CI: 54.3-60.5) months for surgery plus radiation therapy or/and chemotherapy.

As shown in Table 3, the 1-, 3 -, and 5-year OS rate of patients with ESCC who did not receive any treatment were $30.1 \%, 11 \%, 3.6 \%$, while the patients with ESCC who undergo trimodality therapy had a $1-, 3$-, and 5-year OS rate of $80.5 \%, 35.4 \%, 11.6 \%$, respectively. The difference in OS between the two subgroups was significant $(\mathrm{P}<0.001)$. Surgery alone group had better 1-, 3- and 5-year OS than radiation therapy or/and chemotherapy group (OS: $78.5 \%$ vs. $59.2 \%, 37.9 \%$ vs. $18.4 \%, 16.9 \%$ vs. $6.1 \%$ ), the survival differences between subgroups were significant $(\mathrm{P}<0.001)$.

\section{Univariate and Multivariate analysis of survival}

The results of the univariate and multivariate Cox analysis of OS were shown in Table 4. On univariate analysis, survival analysis revealed that increased age ( $\geq 60$ years) and race (black and white) were both significant risk factors for ESCC (HRs $>1$, all $\mathrm{P}<0.05$ ). On multivariable Cox regression, age and therapy were not prognostic factors for ESCC (all $\mathrm{P}>0.05)$. Grade was independent risk factors affecting OS (poor vs. other grades, HR, 1.242, 95\% CI: $1.062-1.454, \mathrm{P}=0.007)$.

\section{Discussion}

The role of surgery alone in the treatment of EC has been recognized as a first-line treatment. In the local advanced EC, the main direction shifts to neoadjuvant chemotherapy before surgery (22-24). In our study, the data shown that the survival prognosis of ESCC was significantly different from the choice of different treatment methods, pure surgery and the triple therapy of surgery, radiotherapy and chemotherapy is superior to radiotherapy and chemotherapy alone in survival and prognosis, with statistical significance.

As for the survival effect of postoperative adjuvant chemotherapy, previous reports have shown that adjuvant chemotherapy can improve disease-free survival rate. However, these reports have shown that there were no significant difference in OS between postoperative adjuvant 
Table 1 Characteristics of patients with esophageal in stage I to III cancer $(n=3,346)$

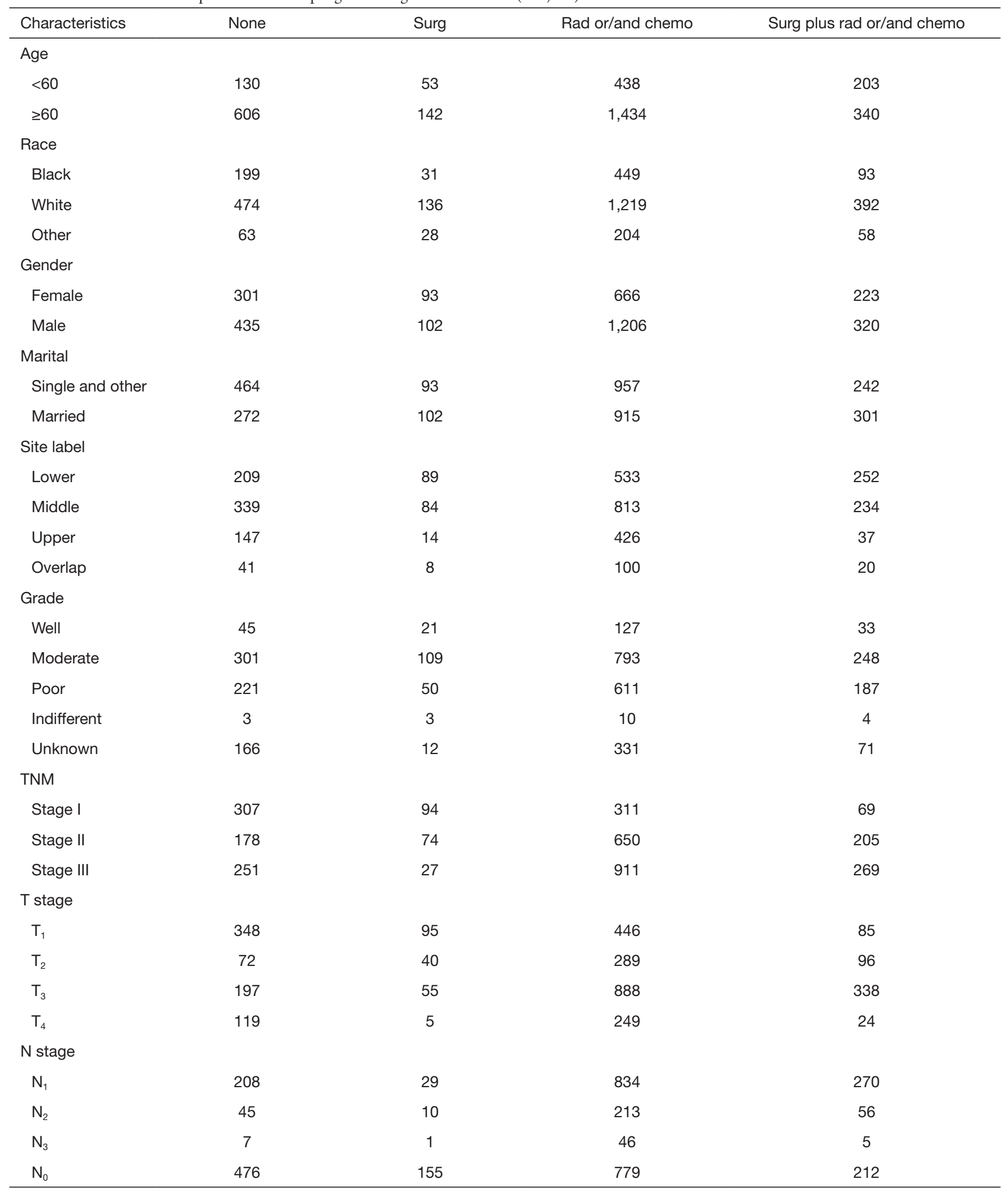

Surg, surgery; rad, radiation; chemo, chemotherapy. 
A

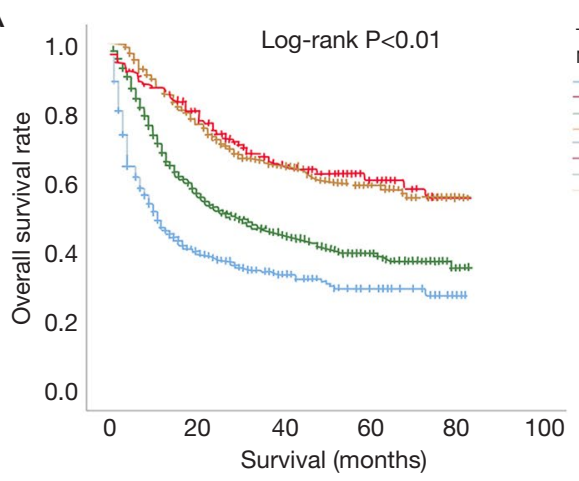

C

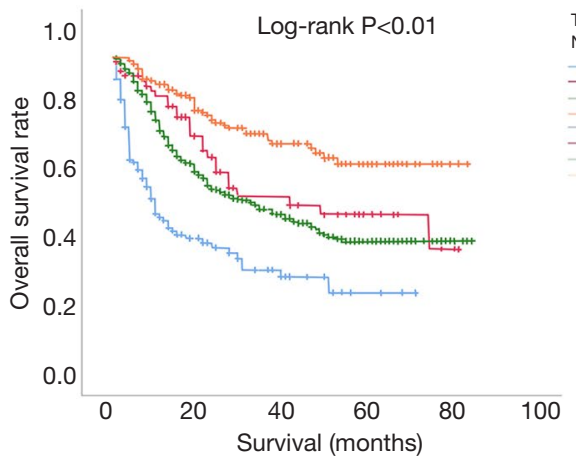

B

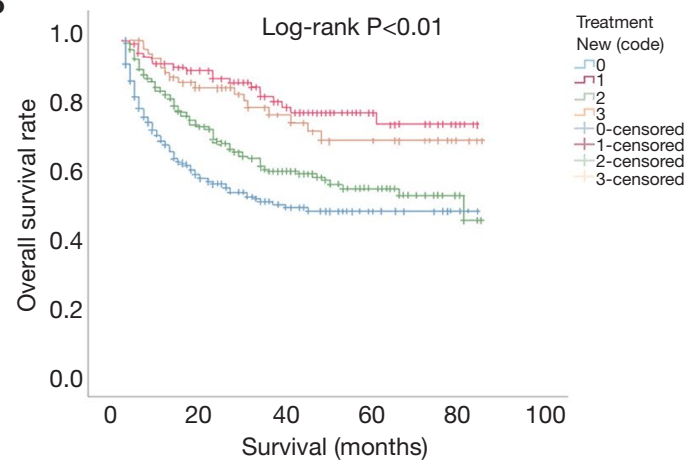

D

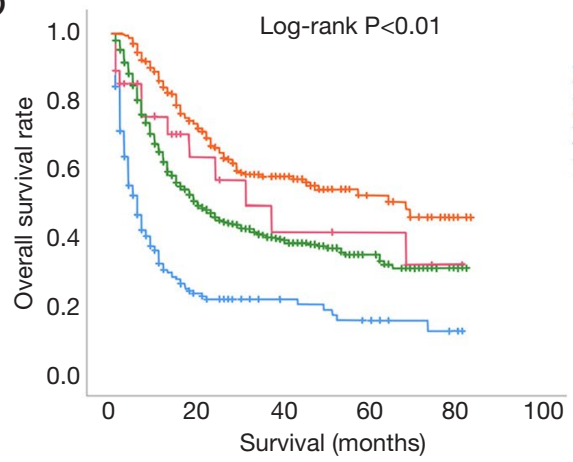

Treatment New (code) reatment ew (code) $\neg 1$
$\neg 2$ - 0 -censored 1-censored 3-censored

Figure 1 OSs according to the treatment modality. (A) Kaplan-Meier survival curves for patients with ESCC on the basis of four treatments. (B,C,D) OS by type of treatment of patients stage I, II and III respectively. 0, 1, 2 and 3 represent for four treatment methods (none, surgery, radiation therapy or/and chemotherapy and surgery plus radiation therapy or/and chemotherapy respectively). ESCC, esophageal squamous cell carcinoma; OS, overall survival.

Table 2 Mean and median survival analysis time

\begin{tabular}{|c|c|c|c|c|c|c|c|c|}
\hline \multirow{2}{*}{$\begin{array}{l}\text { Treatment } \\
\text { new (code) }\end{array}$} & \multicolumn{4}{|c|}{ Mean } & \multicolumn{4}{|c|}{ Median } \\
\hline & $\operatorname{Ref}^{a}$ & SE & Down & Up & $\operatorname{Ref}^{a}$ & SE & Down & Up \\
\hline 0 & 31.711 & 1.608 & 28.558 & 34.863 & 11.000 & 1.234 & 8.581 & 13.419 \\
\hline 1 & 58.141 & 2.546 & 53.150 & 63.131 & - & - & - & - \\
\hline 3 & 57.401 & 1.572 & 54.319 & 60.482 & - & - & - & - \\
\hline All & 44.148 & 0.722 & 42.732 & 45.563 & 34.000 & 2.451 & 29.197 & 38.803 \\
\hline
\end{tabular}

a , If the survival analysis time has been censored, the estimation will be limited to the maximum survival analysis time.

chemotherapy and surgery. Similarly, there were no significant difference in survival and prognosis between pure surgery and trimodality therapy in this study $(8,25,26)$. Besides, through the comparison of different stages of treatment options, the data shows that the survival time in
ESCC patients with stage II/III who had received surgery and trimodality therapy are superior than that in other groups. On the stage II of ESCC patients who treated by trimodality therapy is better than that of other treatments. Therefore, it is beneficial for patients with ESCC in clinical 
Table 3 Survival rate in four treatments

\begin{tabular}{|c|c|c|c|c|}
\hline Treatment & 1 -year survival rate $(\%)$ & 3-year survival rate $(\%)$ & 5 -year survival rate $(\%)$ & $P$ value \\
\hline Surgery [1] & 78.5 & 37.9 & 16.9 & $<0.001$ \\
\hline Radiation therapy or/and chemotherapy [2] & 59.2 & 18.4 & 6.1 & $<0.001$ \\
\hline $\begin{array}{l}\text { Surgery plus radiation therapy or/and } \\
\text { chemotherapy [3] }\end{array}$ & 80.5 & 35.4 & 11.6 & $<0.001$ \\
\hline
\end{tabular}

Table 4 Univariate and multivariate analysis of prognostic factors associated with OS

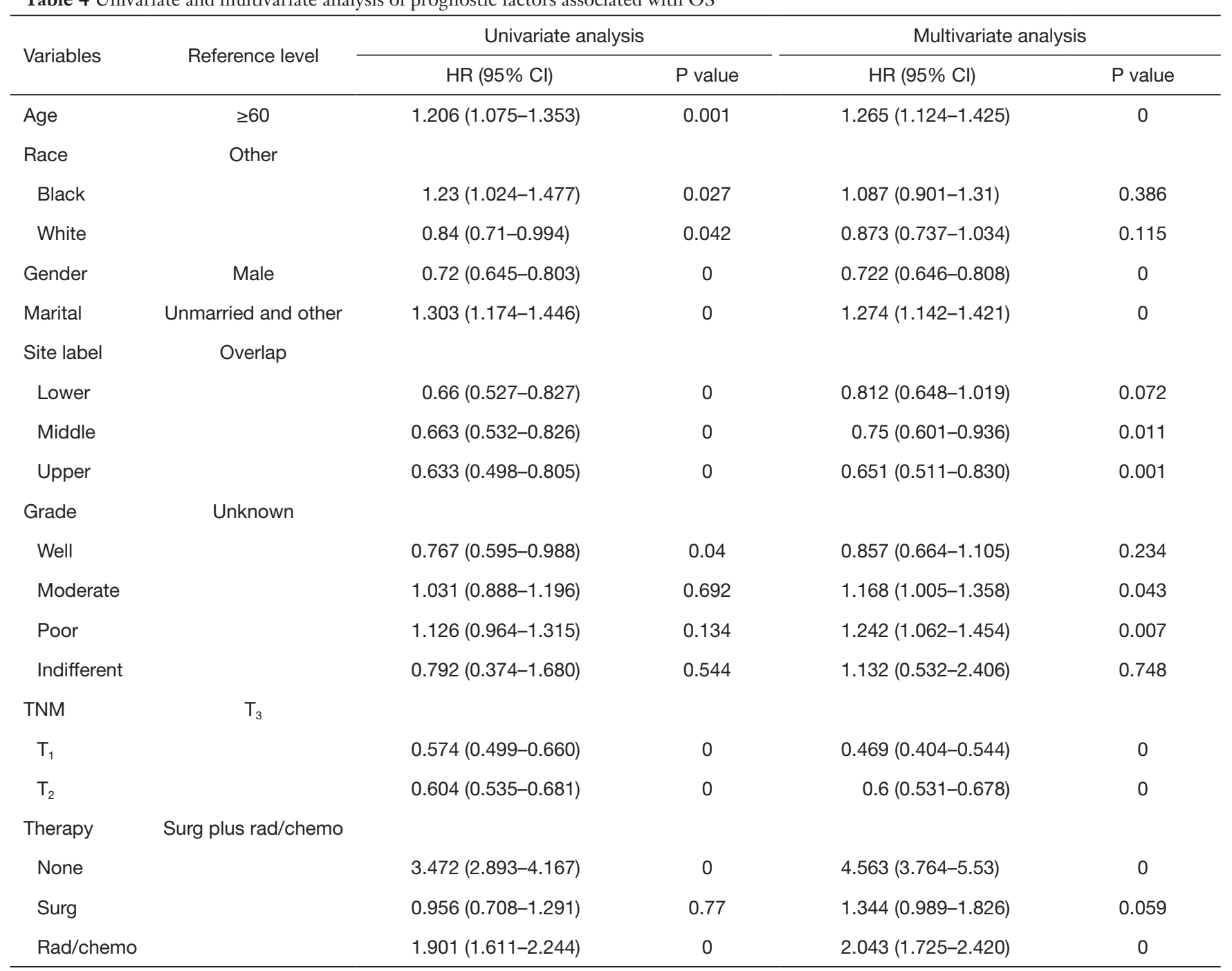

OS, overall survival; surg, surgery; rad, radiation; chemo, chemotherapy.

stage II/III to choose preoperative chemotherapy (27).

Previous studies have shown that the theoretical advantages of combined therapy in the treatment of ESCC including potential tumor downstaging before surgery by reduce tumor volume and micrometastasis. What's more, potential disadvantages including morbidity and mortality by toxicities 
associated with increased antitumor drug selection and dosage, and an eventual delay in valid surgical treatment (28). These data emphasize the importance of thorough preoperative staging in the treatment of ESCC. The important role of neoadjuvant therapy in improving the survival rate of locally advanced ESCC.

Surgical treatment is the standard treatment for early ESCC, but the simple surgical treatment has caused objections (29). The 5-year survival rate of surgery alone was $15-20 \%$ (30). While for the chemoradiotherapy group, the 1-, 3-, and 5-year OS rates were 75.3\%, 35.6\%, and $25.3 \%$, respectively, and $61.5 \%, 26.7 \%$, and $17.6 \%$ for the RT-alone group (31). Therefore, chemotherapy based on radiotherapy has a significant survival meaning $(32,33)$. Multidisciplinary evaluation and treatment have been shown that it can improve the clinical decision-making in ESCC $(34,35)$.

On univariate analysis, age was the prognostic factor of OS $(\mathrm{P}<0.05)$, with elderly patients with ESCC obtaining a more prolonged OS. On multivariate analysis, grade and tumor location was the prognostic factor of OS $(\mathrm{P}<0.05)$. Therefore, we speculated that the effect of age, grade and location are playing an important role on the prognosis of ESCC.

However, for the preoperative radiation dose, chemotherapeutic drugs and specific surgical methods were not unified in the SEER database, there have several deficiencies in our study. We couldn't research the relationship among dose, specific drugs and damage by specific surgical methods.

In conclusion, this work examined the outcomes of different stages of ESCC under different treatment, which has provided a valuable reference for the choice of treatment of ESCC in the future. Although it has not made important contributions to the diagnosis and treatment of ESCC, we still continue to affirm that neoadjuvant radiotherapy and chemotherapy plus surgery are essential. Operation alone can't solve the problem of ESCC forever. At present, we believe that a precise preoperative staging is necessary for the choice of treatment of ESCC. Through the comparison of extensive sample database, this study made precise the outcome of different stages and different treatments of ESCC, which has guiding significance for the choice of treatment of ESCC in the future.

\section{Acknowledgments}

We acknowledged Dr. Jin He for his partial contribution to polish the article.
Funding: None.

\section{Footnote}

Reporting Checklist: The authors have completed the STROBE reporting checklist. Available at http://dx.doi. org/10.21037/tcr-20-2995

Conflicts of Interest: All authors have completed the ICMJE uniform disclosure form (available at http://dx.doi. org/10.21037/tcr-20-2995). The authors have no conflicts of interest to declare.

Ethical Statement: The authors are accountable for all aspects of the work in ensuring that questions related to the accuracy or integrity of any part of the work are appropriately investigated and resolved. The study was conducted in accordance with the Declaration of Helsinki (as revised in 2013).

Open Access Statement: This is an Open Access article distributed in accordance with the Creative Commons Attribution-NonCommercial-NoDerivs 4.0 International License (CC BY-NC-ND 4.0), which permits the noncommercial replication and distribution of the article with the strict proviso that no changes or edits are made and the original work is properly cited (including links to both the formal publication through the relevant DOI and the license). See: https://creativecommons.org/licenses/by-nc-nd/4.0/.

\section{References}

1. Sharma P, Katzka DA, Gupta N, et al. Quality indicators for the management of Barrett's esophagus, dysplasia, and esophageal adenocarcinoma: international consensus recommendations from the American Gastroenterological Association Symposium. Gastroenterology 2015;149:1599-606.

2. Zeng H, Zheng R, Guo Y, et al. Cancer survival in China, 2003-2005: a population-based study. Int J Cancer 2015;136:1921-30.

3. Watanabe M, Otake R, Kozuki R, et al. Correction to: Recent progress in multidisciplinary treatment for patients with esophageal cancer. Surg Today 2020;50:425. Erratum for: Surg Today 2020;50:12-20.

4. Yuan LG, Mao YS. Current status of prognostic evaluation of esophageal cancer patients by circumferential resection margin. Zhonghua Zhong Liu Za Zhi 2019;41:241-5. 
5. Huang L. Updates in version 2.2018 of the NCCN guidelines for esophageal and esophagogastric junction cancers. Oncology and Translational Medicine 2018;4:116-9.

6. Wani S, Qumseya B, Sultan S, et al. Endoscopic eradication therapy for patients with Barrett's esophagusassociated dysplasia and intramucosal cancer. Gastrointest Endosc 2018;87:907-31.e9.

7. Chao YK, Yeh CJ, Lee MH, et al. Factors associated with false-negative endoscopic biopsy results after neoadjuvant chemoradiotherapy in patients with esophageal squamous cell carcinoma. Medicine (Baltimore) 2015;94:e588.

8. Ando N, Iizuka T, Ide H, et al. Surgery plus chemotherapy compared with surgery alone for localized squamous cell carcinoma of the thoracic esophagus: a Japan Clinical Oncology Group Study--JCOG9204. J Clin Oncol 2003;21:4592-6.

9. Smyth EC, Lagergren J, Fitzgerald RC, et al. Oesophageal cancer. Nat Rev Dis Primers 2017;3:17048.

10. Ancona E, Ruol A, Santi S, et al. Only pathologic complete response neoadjuvant chemotherapy improves significantly the long term survival of patients with resectable esophageal squamous cell carcinoma: final report of a randomized. Controlled trial of preoperative chemotherapy versus surgery alone. Cancer 2001;91:2165-74.

11. Meredith KL, Weber JM, Turaga KK, et al. Pathologic response after neoadjuvant therapy is the major determinant of survival in patients with esophageal cancer. Ann Surg Oncol 2010;17:1159-67.

12. Yoon HH, Lewis MA, Shi Q, et al. Prognostic impact of body mass index stratified by smoking status in patients with esophageal adenocarcinoma. J Clin Oncol 2011;29:4561-7.

13. Markar SR, Low DE. Physiology, not chronology, dictates outcomes after esophagectomy for esophageal cancer: outcomes in patients 80 years and older. Ann Surg Oncol 2013;20:1020-6.

14. Mariette C, De Botton ML, Piessen G. Surgery in esophageal and gastric cancer patients: what is the role for nutrition support in your daily practice? Ann Surg Oncol 2012;19:2128-34.

15. Amin RN, Parikh SJ, Reddy GVG, et al. Early esophageal cancer specific survival is unaffected by anatomical location of tumor: a population-based study. Can J Gastroenterol Hepatol 2016;2016:6132640.

16. Nafteux PR, Lerut TE, Villeneuve PJ, et al. Signet ring cells in esophageal and gastroesophageal junction carcinomas have a more aggressive biological behavior. Ann Surg 2014;260:1023-9.

17. Bouvier AM, Binquet C, Gagnaire A, et al. Management and prognosis of esophageal cancers: has progress been made? Eur J Cancer 2006;42:228-33.

18. Nafteux PR, Lerut AM, Moons J, et al. International multicenter study on the impact of extracapsular lymph node involvement in primary surgery adenocarcinoma of the esophagus on overall survival and staging systems. Ann Surg 2015;262:809-15; discussion 815-6.

19. Alattar AA, Brandel MG, Hirshman BR, et al Oligodendroglioma resection: a Surveillance, Epidemiology, and End Results (SEER) analysis. J Neurosurg 2018;128:1076-83.

20. De Neve J, Gerds TA. On the interpretation of the hazard ratio in Cox regression. Biom J 2020;62:742-50.

21. Gao S, Pu N, Liu L, et al. The latest exploration of staging and prognostic classification for pancreatic neuroendocrine tumors: a large population-based study. J Cancer 2018;9:1698-706.

22. Ajani JA, D'Amico TA, Almhanna K, et al. Esophageal and esophagogastric junction cancers, version 1.2015. J Natl Compr Canc Netw 2015;13:194-227.

23. Mariette C, Robb WB, Piessen G, et al. Neoadjuvant chemoradiation in oesophageal cancer. Lancet Oncol 2015;16:1008-9.

24. Wang BY, Lin PY, Wu SC, et al. Comparison of pathologic stage in patients receiving esophagectomy with and without preoperative chemoradiation therapy for esophageal SCC. J Natl Compr Canc Netw 2014;12:1697-705.

25. Zhang SS, Yang H, Xie X, et al. Adjuvant chemotherapy versus surgery alone for esophageal squamous cell carcinoma: a meta-analysis of randomized controlled trials and nonrandomized studies. Dis Esophagus 2014;27:574-84.

26. Qin RQ, Wen YS, Wang WP, et al. The role of postoperative adjuvant chemotherapy for lymph nodepositive esophageal squamous cell carcinoma: a propensity score matching analysis. Med Oncol 2016;33:31.

27. Ando N, Kato H, Igaki $\mathrm{H}$, et al. A randomized trial comparing postoperative adjuvant chemotherapy with cisplatin and 5 -fluorouracil versus preoperative chemotherapy for localized advanced squamous cell carcinoma of the thoracic esophagus (JCOG9907). Ann Surg Oncol 2012;19:68-74.

28. Kato H, Nakajima M. Treatments for esophageal cancer: a review. Gen Thorac Cardiovasc Surg 2013;61:330-5. 
29. O'Reilly S, Forastiere AA. Is surgery necessary with multimodality treatment of oesophageal cancer. Ann Oncol 1995;6:519-21.

30. Allum WH, Stenning SP, Bancewicz J, et al. Long-term results of a randomized trial of surgery with or without preoperative chemotherapy in esophageal cancer. J Clin Oncol 2009;27:5062-7.

31. Chen H, Zhou L, Yang Y, et al. Clinical effect of radiotherapy combined with chemotherapy for nonsurgical treatment of the esophageal squamous cell carcinoma. Med Sci Monit 2018;24:4183-91.

32. Wobbes T, Baron B, Paillot B, et al. Prospective randomised study of split-course radiotherapy versus cisplatin plus split-course radiotherapy in inoperable squamous cell carcinoma of the oesophagus. Eur J Cancer 2001;37:470-7.

33. Le Prise E, Etienne PL, Meunier B, et al. A randomized study of chemotherapy, radiation therapy, and surgery versus surgery for localized squamous cell carcinoma of the esophagus. Cancer 1994;73:1779-84.

34. Stahl M, Mariette C, Haustermans K, et al. Oesophageal cancer: ESMO Clinical Practice Guidelines for diagnosis, treatment and follow-up. Ann Oncol 2013;24 Suppl 6:vi51-6.

35. Wang Y, Zhang S, Li F, et al. Therapeutic target database 2020: enriched resource for facilitating research and early development of targeted therapeutics. Nucleic Acids Res 2020;48:D1031-41.
Cite this article as: $\mathrm{Wu} \mathrm{J}$, Yang J, Lin X, Lin L, Jiang W, Xie C. Survival outcomes for patients with four treatments in stages I-III esophageal squamous cell carcinoma: a SEER analysis. Transl Cancer Res 2021;10(5):2144-2152. doi: $10.21037 /$ tcr-20-2995 